\title{
Dissociation of Individual Isotopic Peaks: Predicting Isotopic Distributions of Product Ions in $\mathbf{M S}^{\mathbf{n}}$
}

\author{
Alan L. Rockwood, Mark M. Kushnir, and Gordon J. Nelson \\ ARUP Institute for Clinical and Experimental Pathology, Salt Lake City, Utah, USA
}

\begin{abstract}
Traditional practice in tandem mass spectrometry is to select the mono-isotopic ion for dissociation. However, high molecular weight compounds often have weak mono-isotopic peaks, which limit that approach. Furthermore, the traditional approach does not take advantage of the very rich store of information available in the isotopic patterns from the dissociation of individual non-mono-isotopic peaks. Interpretation of these isotopic patterns requires a theory capable of predicting the patterns. However, a general theory for the prediction of these patterns has been lacking. This paper shows that the patterns can be obtained from a certain vector product, the outer product, of the full isotopic distribution of the product ion with the full isotopic distribution of the complementary product. Unlike previous approaches, the method is applicable to systems of arbitrary isotopic complexity. The patterns are potentially useful for elucidation of dissociation pathways, elemental composition, and chemical structure. The paper presents several applications of the theory. (J Am Soc Mass Spectrom 2003, 14, 311-322) (C) 2003 American Society for Mass Spectrometry
\end{abstract}

$\mathrm{T}$ Traditional practice in tandem mass spectrometry is to select the mono-isotopic ion for dissociation $[1,2]$. This simplifies interpretation by eliminating any complicating isotopic patterns. This approach works well for most low molecular weight compounds. However, high molecular weight compounds often have weak mono-isotopic peaks. In such cases sensitivity may be improved by selecting a different isotopic peak for dissociation.

Furthermore, the very fact that isotopic patterns from the dissociation of non-mono-isotopic peaks are more complex than those from the dissociation of mono-isotopic peaks means that they are informationrich and potentially useful for elucidation of chemical composition and structure. The traditional approach of utilizing only the mono-isotopic peak does not take advantage of this rich store of information. It has long been recognized that the isotopic patterns of product ions from the dissociation of non-mono-isotopic peaks contain valuable information about molecular composition and structure [3-10]. Despite an early recognition of the value of this type of experiment, the technique of tandem mass spectrometry of selected non-mono-isotopic peaks has not been widely adopted.

One factor that may have prevented a wider acceptance of the technique is the absence of a general theory

Published online March 10, 2003

Address reprint requests to Dr. A. L. Rockwood, ARUP Institute for Clinical and Experimental Pathology, 500 Chipeta Way, Salt Lake City, UT 84108,

USA. E-mail: rockwoal@aruplab.com of such fragmentation and a consequent difficulty in interpretation of the isotopic patterns. Previous theories have been based on simplified treatments that are not rigorously applicable to the general case [3-5]. For example, the discussion of $\mathrm{O}^{\prime}$ Connor et al. was based on assumptions that the chemical elements in the formula each contain only two isotopes, and that the elemental isotopes are spaced one Da apart [5]. However more than $40 \%$ of the elements found in nature contain three or more isotopes, including such common elements as oxygen (three isotopes), sulfur (four isotopes), iron (four isotopes), and calcium (six isotopes). Consequently, a general theory is needed for the calculation of the isotopic distributions resulting from the dissociation of single isotopic peaks.

In this paper we present a general approach to the problem of predicting the isotopic patterns resulting from the dissociation of single isotopic peaks. Also presented are experimental comparisons to the theoretical results.

\section{Concepts and Definitions}

Throughout the paper the terms "ion" and "peak" are often used interchangeably. This paper deals exclusively with random isotopic distributions. This rules out cases in which a significant isotope effect exists. It also rules out cases in which there is an unknown bias in the relative abundance of isotopes at different positions within a molecule. When an isotope effect is significant, the algorithm presented here can be consid- 
ered an approximate method rather than an exact method. One would normally expect isotope effects to be insignificant except in cases involving hydrogen and deuterium.

The algorithm makes extensive use of vectors and matrices. Unless specified otherwise, all vectors in this paper are column vectors. We make a slight departure from conventional vector and matrix notation to allow their indices to start at zero rather than one. The indices of the vectors and matrices are identified with integer masses. Utilization of zero indices (i.e., zero masses) allows simplification and generalization of some formulas in the paper and is not meant to suggest that zero masses are physically meaningful for molecules.

This paper refers to "reverse diagonal bands" of matrices. A reverse diagonal band is composed of a series of matrix elements read out in a specific order, starting at a selected matrix element on the left column, then reading out the reverse diagonal matrix elements sequentially. Each successive element of the readout occupies a position one column to the right and run row up from the previous element.

There are two kinds of isotopic distributions discussed in this paper. The first is the result of the dissociation of the full isotopic distribution of the parent ion. This distribution will be referred to as the "full" isotopic distribution of the product ion, to distinguish it from distributions resulting from the dissociation of individual isotopic peaks. Experimentally, the full isotopic distribution of the product ion would be observed in a tandem mass spectrometry experiment in which the parent ion resolution was low enough to pass the full parent ion distribution without mass discrimination. This is also the distribution that one would observe if the product ion were formed in the ion source rather than in a dissociation cell of a tandem mass spectrometer. Furthermore, it is the distribution one would calculate from the chemical formula of the product ion using standard theoretical methods [11-18]. The other type of isotopic distribution is the result of the dissociation of an individual isotopic peak of the parent ion.

The isotopic distribution of the parent ion will be represented by $h$, which is a discrete probability distribution expressed as a vector. The elements of this vector add to unity. The relative intensities of the individual isotopic peaks of $h$ are represented by $h(n)$, where $n$ is the vector index. The parameter $n$ also represents the number of nucleons present in the isotopic peak, which we will sometimes call "integer mass".

The value of the first non-zero element of $h$ is the abundance of the mono-isotopic peak of the parent ion. However, in a formal sense this will not be the first element of the vector. For example, consider $h(0)$ for $\mathrm{NH}_{6} \mathrm{O}^{+}$. There is no isotopic peak at mass zero, so the value of $h(0)$ is zero. The first nonzero peak for $\mathrm{NH}_{6} \mathrm{O}^{+}$ has a mass of 36, so the first non-zero element of $h$ is $h(36)$. It has a value of 0.9934 , representing a probability of 0.9934 . The integer mass of this, the mono-isotopic, peak is 36 and the true mass is 36.004 . At times it is convenient in this paper to use $h$ as an identifying symbol for the parent ion. Therefore the same symbol is given two different meanings, a slight looseness of nomenclature clarified by the context in which it will be used.

Consider the dissociation of a parent ion via a single dissociation pathway into two dissociation products. The two dissociation products have well-defined chemical compositions. These compositions are complementary in the sense that the chemical formulas of the two dissociation products, taken together, equal the chemical formula of the parent ion. One of these products will be designated as the "product ion" and the second will be designated as the "complementary product." For example, the ion $\mathrm{NH}_{6} \mathrm{O}^{+}$could dissociate into a product ion, $\mathrm{NH}_{4}^{+}$, and a complementary product, $\mathrm{H}_{2} \mathrm{O}$. The full isotopic profiles of the product ion and the complementary product are vectors $g$ and $f$, respectively, making them analogous to vector $h$. We also use $g$ and $f$ loosely as identifiers for the product ion and complementary product. Introducing this slight ambiguity avoids a proliferation of symbols, and the context in which these symbols are used will resolve any ambiguity.

We will initially assume that one knows the elemental compositions of the parent and product ions. In most applications, "knowing" a composition means that the composition is hypothesized and the calculation is then based on this hypothesis.

The algorithm does not distinguish between charged and uncharged species. Hence, the "parent ion" and the "product ion" could be either charged or uncharged, and the roles of the product ion and complementary product can be interchanged.

Wherever the symbol $\mathrm{M}^{+}$appears in this paper it refers to the mono-isotopic form of the ion actually detected in the mass spectrometer, including any charge carrying adduct which may be attached, such as a proton or ammonium adduct. Symbols such as $(\mathrm{M}+$ $1)^{+},(\mathrm{M}+2)^{+}$, etc. refer to successively higher isotopic peaks.

\section{The Algorithm}

Consider the two-dimensional matrix, $C$, whose elements are defined by the following mathematical product:

$$
C(i, j)=g(i) f(j)
$$

Eq 1 is defines the outer product of $g$ and $f$ [19]. Let us define three additional matrices, $D, E$, and $F$.

$$
\begin{aligned}
& D(i, j)=i \\
& E(i, j)=i+j \\
& F(i, j)=j
\end{aligned}
$$


Let $k$ be the integer mass of an isotopic peak selected for dissociation. For each value of $k$ there is a reverse diagonal band of $E$ whose elements equal the integer mass, $k$. There is a corresponding set of elements of matrix $D$ whose elements are the product ion integer masses that result from the dissociation of parent ion with isotopic mass $k$. For example, if the parent ion mass is given by $k=11$, then the possible masses for the product ion are $11,10,9, \ldots 0$. Elements of matrix $F$ are the masses of the complementary products.

In order to perform the calculation one selects a parent ion of isotopic mass $k$, then generates the following set of ordered pairs:

$$
[D(k-n, n), C(k-n, n)]
$$

where $n$ is an integer dummy variable that runs from $k$ to 0 . The ordered pairs are the mass/intensity pairs for the product ion from the dissociation of the selected isotopic peak of the parent ion. Equations 1, 2, and 5 define the algorithm for product ion scans.

The present paper uses a matrix-based formulation. However, it is possible to ignore the connection to matrices and develop a matrix-less formulation. Let $m_{i}$ represent mass of the ith isotope of the product of dissociation, and $n_{j}$ represent the mass of the $j$ th isotope of the complementary product. Let $I_{i}$ represent the isotopic abundance of the ith isotope of the dissociation product, and let $J_{j}$ represent the isotopic abundance of the $j$ th isotopic peak of the complementary product. $I_{i}$ and $J_{j}$ are not taken to mean the abundance resulting from the dissociation of single isotopic peaks, but instead are taken from the full isotopic profiles, as discussed previously. Assume that we are to dissociate a parent ion isotopic peak of mass $o_{k}$. The abundance of the product ion isotopic peak of mass $m_{i}$ from this dissociation is $I_{i} \cdot J_{j}$, subject to the restriction that $n_{j}=$ $o_{k}-m_{i}$, where the symbol $\cdot$ denotes ordinary multiplication.

Although a matrix-less description of the algorithm is possible, a matrix-based formulation facilitates a proof of the algorithm, makes it easy to express related quantities, provides a flexible and uniform notation, provides a simple way to describe several different scan functions, and makes it easy to translate the algorithm into computer code. Therefore, the rest of the paper continues with the matrix-based approach.

\section{Why It Works}

One can combine the chemical formulas of two compounds to form a compound of higher molecular weight. When one combines two molecular pieces to form a larger molecule, the isotopic profile of the resultant molecule is the convolution of the isotopic profiles of the two pieces that are being combined. In the present case, this corresponds to generation of the isotopic profile of the parent ion, $h$, starting with the isotopic profiles of the product ion, $f$, and complementary product, $g$. As part of this process one calculates the matrix elements of $C$.

The matrix $C$ is a "convolution matrix" because it contains all the terms required to calculate the convolution of $f$ with $g$. The convolution process is completed by applying any of the following formulas:

$$
\begin{aligned}
h(k) & =\sum_{i} C(i,(k-i))=\sum_{j} C((k-j), j) \\
& =\sum_{i} g(i) f(k-i)=\sum_{j} g(k-j) f(j)
\end{aligned}
$$

or

$$
h(k)=\sum_{i, j} C(i, j)=\sum_{i, j} g(i) f(j), k=i+j
$$

These equations state several equivalent forms for the well-known formulas for convolution of discrete functions [20]. The same matrix, $C$, is also a "dissociation matrix" because it contains all the information relating to intensities of product ion, and complementary product formed by the dissociation of the parent.

Matrix $C$ represents all the different combinations of isotopic peaks of $f$ and $g$ that contribute mathematically to the isotopic peaks of $h$. For example, the monoisotopic peak of $\mathrm{NH}_{6} \mathrm{O}^{+}$contains 36 nucleons. There is only one combination of peaks of $\mathrm{H}_{2} \mathrm{O}$ and $\mathrm{NH}_{4}^{+}$that contribute a non-zero intensity, namely the $\mathrm{H}_{2} \mathrm{O}$ with 18 nucleons [which we will designate as ${ }^{18}\left(\mathrm{H}_{2} \mathrm{O}\right)$ ] and the $\mathrm{NH}_{4}^{+}$with 18 nucleons [which we will designate as $\left.{ }^{18}\left(\mathrm{NH}_{4}^{+}\right)\right]$. There are two combinations that contribute to ${ }^{37}\left(\mathrm{NH}_{6} \mathrm{O}^{+}\right)$, the first combination being ${ }^{18}\left(\mathrm{H}_{2} \mathrm{O}\right)+$ ${ }^{19}\left(\mathrm{NH}_{4}^{+}\right)$, and the second combination being ${ }^{19}\left(\mathrm{H}_{2} \mathrm{O}\right)+$ ${ }^{18}\left(\mathrm{NH}_{4}^{+}\right)$. There are three ways to generate the ${ }^{38}\left(\mathrm{NH}_{6} \mathrm{O}^{+}\right)$, four ways to generate ${ }^{39}\left(\mathrm{NH}_{6} \mathrm{O}^{+}\right)$, and so forth. The elements of matrix $C$ contain the relative contribution that each individual combination makes to the parent ion.

One can think of matrix $C$ as being a giant bookkeeping device for isotopic probabilities. Each element of $C$ contains a slice of probability relating to an isotopic composition. Hence, the matrix $C$ can also be named a "probability matrix". The relationships between probabilities and isotopic compositions are best understood by referring to Figure 1. Figure 1a is an "isotopic composition matrix" showing all the combinations of isotopic peaks of $\mathrm{NH}_{4}^{+}$and $\mathrm{H}_{2} \mathrm{O}$, such as outlined in the previous paragraph. For convenience, only the significant entries of the composition matrix are shown. For example, the combination $\left[{ }^{17}\left(\mathrm{NH}_{4}^{+}\right)+{ }^{16}\left(\mathrm{H}_{2} \mathrm{O}\right)\right]$ is not shown because there is no naturally occurring ${ }^{16}\left(\mathrm{H}_{2} \mathrm{O}\right)$. The intensity of the $\left[{ }^{21}\left(\mathrm{NH}_{4}^{+}\right)+{ }^{22}\left(\mathrm{H}_{2} \mathrm{O}\right)\right]$ combination is so small as to be negligible, so it is also not shown. Figure $1 \mathrm{~b}$ shows a related matrix for the elemental formula $\mathrm{NH}_{6} \mathrm{O}^{+}$, which is the result of combining $\mathrm{NH}_{4}^{+}$ and $\mathrm{H}_{2} \mathrm{O}$. Figure $1 \mathrm{c}$ shows a matrix of integer molecular 


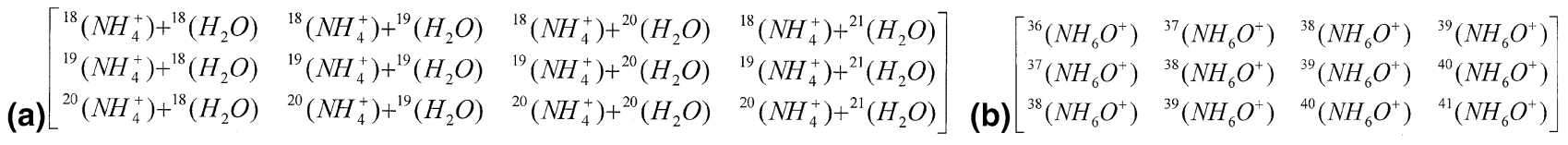
(c) $\left[\begin{array}{llll}36 & 37 & 38 & 39 \\ 37 & 38 & 39 & 40 \\ 38 & 39 & 40 & 41\end{array}\right]$
$[(0.995739,0.997291)$
$(0.004258,0.997291)$
$(0.995739,0.000673)$
$(0.995739,0.002036)$
$(0.995739,0.000001)$
(d) $[(0.000002,0.997291)$
$(0.004258,0.000673)$
$(0.004258,0.002036)$
$(0.004258,0.000001)$
$(0.000002,0.000673)$
$(0.000002,0.002036)$
$(0.000002,0.000001)$

$\left[\begin{array}{llll}0.995739 *(0.997291) & 0.995739 *(0.000673) & 0.995739 *(0.002036) & 0.995739 *(0.000001) \\ 0.004258 *(0.997291) & 0.004258 *(0.000673) & 0.004258 *(0.002036) & 0.004258 *(0.000001) \\ 0.000002 *(0.997291) & 0.000002 *(0.000673) & 0.000002 *(0.002036) & 0.000002 *(0.000001)\end{array}\right] \quad$ (f) $\left[\begin{array}{llll}0.993042 & 0.000670 & 0.002027 & 0.000001 \\ 0.004246 & 0.000003 & 0.000009 & 0.000000 \\ 0.000002 & 0.000000 & 0.000000 & 0.000000\end{array}\right]$

(e) $\left[\begin{array}{llll}0.000002 *(0.997291) & 0.000002 *(0.000673) & 0.000002 *(0.002036) & 0.000002 *(0.000001)\end{array}\right] \quad(\mathbf{f})\left[\begin{array}{lll}0.000002 & 0.000000 & 0.00000\end{array}\right.$
Figure 1. Matrices illustrating the relationships between the outer product, full isotopic profiles of product ion and complementary product, and isotopic profiles of product ions from dissociation of individual isotopic peaks of parent ion. Only entries for isotopic peaks of significant intensity are shown. Hence, the upper-left-most element of each matrix has vertical and horizontal indices of 18 and 18, respectively. (a) Isotopic composition matrix showing how different combinations of isotopic peaks of product ion and complementary product combine to form individual isotopic peaks of parent ion, isotopic masses shown as upper-left superscripts, (b) resulting combinations of the entries in Figure 1a, isotopic masses shown as upper-left superscript, (c) isotopic masses of parent ions from the various combinations represented in Figure 1a, values taken from the upper-left superscripts of Figure $1 \mathrm{~b}$, (d) probabilities of isotopic peaks of product ion and complementary product, taken from full isotopic distributions of parent and product, expressed as ordered pairs, (e) multiplications formed by the ordered pairs from Figure $1 \mathrm{~d}$, (f) the $C$-matrix for the convolution process and the dissociation process obtained by multiplications indicated in Figure 1e.

masses corresponding to combinations illustrated in Figure $1 \mathrm{a}$ and $\mathrm{b}$. The values in Figure $1 \mathrm{c}$ are the superscripts of the corresponding cells in Figure 1b. The matrix in Figure 1c is matrix $E$ for the dissociation of $\mathrm{NH}_{6} \mathrm{O}^{+}$, forming $\mathrm{NH}_{4}^{+}$and $\mathrm{H}_{2} \mathrm{O}$. Figure $1 \mathrm{~d}$ shows the probability factors taken from the full isotopic distributions of $\mathrm{NH}_{4}^{+}$and $\mathrm{H}_{2} \mathrm{O}$. These entries are represented as ordered pairs. Unlike the ordered pairs discussed previously, both elements of these ordered pairs are probabilities. Figure 1e shows how these ordered pairs are multiplied to generate matrix $C$. Figure $1 \mathrm{f}$ shows final result of the multiplication operations specified in Figure 1e. This is matrix $C$ for the dissociation of $\mathrm{NH}_{6} \mathrm{O}^{+}$forming $\mathrm{NH}_{4}^{+}$and $\mathrm{H}_{2} \mathrm{O}$.

Each of the numbers in Figure $1 \mathrm{f}$ is a probability that relates back to an isotopic composition specified in a corresponding cell of 1 a. For example, $C(19,18)$ corresponds to composition ${ }^{19}\left(\mathrm{NH}_{4}^{+}\right)+{ }^{18}\left(\mathrm{H}_{2} \mathrm{O}\right)$. It is the probability for forming the specified combination and it has a value of 0.0043 . In other words ${ }^{19}\left(\mathrm{NH}_{4}^{+}\right)+{ }^{18}\left(\mathrm{H}_{2} \mathrm{O}\right)$ contributes $0.43 \%$ to the full isotopic profile of $\mathrm{NH}_{6} \mathrm{O}^{+}$. Analogously, $C(18,19)$ corresponds to a composition of ${ }^{18}\left(\mathrm{NH}_{4}^{+}\right)+{ }^{19}\left(\mathrm{H}_{2} \mathrm{O}\right)$ and has a value of 0.0007 , so it contributes $0.07 \%$ to the full isotopic profile of $\mathrm{NH}_{6} \mathrm{O}^{+}$. Cells $C(19,18)$ and $C(18,19)$ both correspond to an integer mass (i.e., nucleon number) of 37 , but they refer to different ways of generating an ${ }^{37}\left(\mathrm{NH}_{6} \mathrm{O}^{+}\right)$, so they are distinct quantities.

So far we have been looking at $C$ from the perspective of building the isotopic distribution of $h$ starting with the natural isotopic distributions of $f$ and $g$. In other words, we have assembled the parent ion from the product ion and the complementary product. Now, let us look at matrix $C$ from the reverse perspective, that of dissociation. Because there was only one way to form the monoisotopic peak (mass $36 \mathrm{Da}$ ), there must be only one way to dissociate the monoisotopic peak of $h$, which is to reverse the process used to combine the peaks. This means that dissociation of this peak yields ${ }^{18}\left(\mathrm{H}_{2} \mathrm{O}\right)$ and ${ }^{18}\left(\mathrm{NH}_{4}^{+}\right)$, so $\mathrm{NH}_{4}^{+}$will appear as a single peak at $\mathrm{m} / \mathrm{z} 18$ in the mass spectrum.

The next parent ion peak contains 37 nucleons. There are two ways to build this, starting from $\mathrm{H}_{2} \mathrm{O}$ and $\mathrm{NH}_{4}^{+}$. One is from ${ }^{18}\left(\mathrm{H}_{2} \mathrm{O}\right)+{ }^{19}\left(\mathrm{NH}_{4}^{+}\right)$, and the other is from ${ }^{19}\left(\mathrm{H}_{2} \mathrm{O}\right)+{ }^{18}\left(\mathrm{NH}_{4}^{+}\right)$. We can therefore divide the ${ }^{37}\left(\mathrm{NH}_{6} \mathrm{O}^{+}\right)$ions into two mutually exclusive sub-groups, one whose composition is ${ }^{18}\left(\mathrm{H}_{2} \mathrm{O}\right)+$ ${ }^{19}\left(\mathrm{NH}_{4}^{+}\right)$and another whose composition is ${ }^{19}\left(\mathrm{H}_{2} \mathrm{O}\right)+$ ${ }^{18}\left(\mathrm{NH}_{4}^{+}\right)$. If we take the ${ }^{37}\left(\mathrm{NH}_{6} \mathrm{O}^{+}\right)$peak apart into $\mathrm{H}_{2} \mathrm{O}$ and $\mathrm{NH}_{4}^{+}$then the products must be generated in the same relative abundance as they contributed to ${ }^{37}\left(\mathrm{NH}_{6} \mathrm{O}^{+}\right)$. As mentioned previously, the ${ }^{18}\left(\mathrm{H}_{2} \mathrm{O}\right)+$ ${ }^{19}\left(\mathrm{NH}_{4}^{+}\right)$pair contributes a relative abundance of $0.43 \%$, and the ${ }^{19}\left(\mathrm{H}_{2} \mathrm{O}\right)+{ }^{18}\left(\mathrm{NH}_{4}^{+}\right)$pair contributes a relative abundance of $0.07 \%$. There are no other contributors to the mass 37 parent ion peak. Therefore, the branching ratio for the two dissociation pathways for dissociation of the mass 37 parent ion peak must be (0.43):(0.07), or roughly 6:1. Consequently, in the mass spectrometer one would detect $\mathrm{NH}_{4}^{+}$at masses 19 and 18 at a relative abundance ratio of 6:1.

The pattern continues with the higher isotopic peaks. For example, there are three ways to make the mass 38 peak, four ways to make the mass 39 peak and so forth. Their relative probabilities are read from the appropriate reverse diagonal band of matrix $C$. 


\section{Additional Relationships}

Matrix $C$ contains a wealth of information about the isotopic profiles of parent ions and dissociation products. For example, summing down columns generates $f$, the normal isotopic profile of the complementary product. This can be seen from the following:

$$
\sum_{i} C(i, j)=\sum_{i} g(i) f(j)=f(j) \sum_{i} g(i)=f(j)
$$

where we have used

$$
\sum_{i} g(i)=1
$$

Summing across the rows generates $g$, the normal isotopic profile of the product ion:

$$
\sum_{j} C(i, j)=\sum_{j} g(i) f(j)=f(i) \sum_{i} f(j)=g(i)
$$

where we have used

$$
\sum_{i} f(i)=1
$$

A sum of the reverse diagonal bands generates vector $h$, which is the isotopic distribution of the parent ion. This corresponds to the operation specified in eqs 6 or 7 , the convolution of $f$ with $g$. The matrix elements in a selected row represent the relative intensities of a precursor ion scan. The associated product ion mass is found in the corresponding row of matrix $D$, and the parent ion mass is found in the corresponding row of matrix $E$. The columns of matrix $C$ represent predicted results for constant neutral loss scans. The mass of the neutral loss is given by the corresponding elements of matrix $F$. Matrix $C$ provides a convenient way to calculate the isotopic profiles for any scan function.

\section{Experimental}

\section{Chemicals, Standards, and Solutions}

Succinic acid (SA), ammonium formate, and methanol were purchased from Sigma (St. Louis, MO). 32-desmethoxysirolimus (DMS) was as a gift from Wyeth-Ayerst Research (Princeton, NJ). The di-n-butyl ester of succinic acid (ESA) was prepared by transferring $0.4 \mu \mathrm{mol}$ of the acid standard into a glass tube, the solvent was evaporated, and the residue reconstituted with $50 \mu \mathrm{L}$ of n-butanol containing $3 \mathrm{M} \mathrm{HCl}$. The tube was incubated at $60^{\circ} \mathrm{C}$ for $15 \mathrm{~min}$. Excess derivatizing reagent was evaporated and the remaining residue was reconstituted with $5 \mathrm{~mL}$ of methanol containing $50 \% 0.005 \mathrm{M}$ ammonium formate, $\mathrm{pH}$ 6.5. DMS solution was prepared at a concentration of $5.7 \mu \mathrm{mol} / \mathrm{L}$ in methanol containing 50\% 0.005M ammonium formate, $\mathrm{pH}$ 6.5. The samples were analyzed by syringe infusion with flow rate of $10 \mu \mathrm{L} / \mathrm{min}$ into the TurbolonSpray (TIS) ion source.

\section{Apparatus}

An API 3000 (Applied Biosystems/MDS SCIEX, Foster City, CA) tandem mass spectrometer equipped with syringe pump (Fisher Scientific, Pittsburg, PA) was used in the positive ion mode with TurboIonSpray (TIS) interface. Conditions for the instrumental analysis were as follows. For the single MS experiments the TIS capillary voltage was $5.0 \mathrm{kV}$, the orifice voltage was 15 $\mathrm{V}$ for ESA, and $41 \mathrm{~V}$ for DMR. For the MS/MS experiment the collision energy and collision cell exit potential were 15 and $6 \mathrm{~V}$ for ESA, and 25 and $32 \mathrm{~V}$, for DMR, respectively. The collision gas was nitrogen with a cell pressure of 1.1 Pascal. Dwell time per ion utilized for data acquisition in the experiment was $250 \mathrm{~ms}$. Resolution of the first resolving quadruple (Q1) was set to 0.5 $\mathrm{Da}$ at $50 \%$ height. This produced sufficient sensitivity and allowed elimination of interference from the neighboring natural isotopic analogs separated by $1 \mathrm{Da}$ for the product ions scan experiments. The resolution of the second resolving quadruple (Q3) was set to $0.7 \mathrm{Da}$ at $50 \%$ height to assure sufficient sensitivity for the MRM experiment. Data were acquired from a total of twenty scans. To assure reproducibility of ion intensity ratios, experiments were repeated during three days with three measurements performed per day.

The data for ESA were acquired in product ion scan mode. The following parent ions and product ion scan ranges of the Q3 quadruple were utilized for the ESA: $(m / z): 231+n \rightarrow 175 \pm 5 \mathrm{Da}, 231+n \rightarrow 157 \pm 5 \mathrm{Da}$, and $231+n \rightarrow 101 \pm 5 \mathrm{Da}$; where $n=0$ corresponds to a monoisotopic parent ion and $n=1,2,3 \ldots$ corresponds to higher isotopic peaks. The parent ion corresponds to protonated ESA molecule, $\mathrm{C}_{12} \mathrm{H}_{23} \mathrm{O}_{4}^{+}$.

The data for DMS were obtained in precursor ion scan mode. The chemical composition of DMS is $\mathrm{C}_{50} \mathrm{H}_{77} \mathrm{NO}_{12}$. Tandem mass spectrometry was performed on the ammoniated DMS ion, $\mathrm{C}_{50} \mathrm{H}_{81} \mathrm{~N}_{2} \mathrm{O}_{12}^{+}(\mathrm{m} / \mathrm{z}$ 901). The precursor ions and product ion scan ranges monitored for an ammonium adduct of DMS were $(m / z): 901+n \rightarrow 852 \pm 5 \mathrm{Da}, 901+n \rightarrow 834 \pm 5 \mathrm{Da}$, and $901+n \rightarrow 816 \pm 5 \mathrm{Da}$, where $n=0$ corresponds to a monoisotopic ion and $n=1,2,3 \ldots$ corresponds to higher-mass isotopic peaks.

\section{Results and Discussion}

\section{DMS Precursor Ion Scans}

Figure 2 shows the results for precursor ion scans of DMS. The parent ion for DMS is the ammoniated ion, $\mathrm{C}_{50} \mathrm{H}_{81} \mathrm{~N}_{2} \mathrm{O}_{12}^{+}$. Figure 3 shows the comparison between theoretical and experimental results for precursor ion scans of the $\mathrm{m} / \mathrm{z} 834$ product ion of DMS. Two different scales are shown in order to compare both the large and 


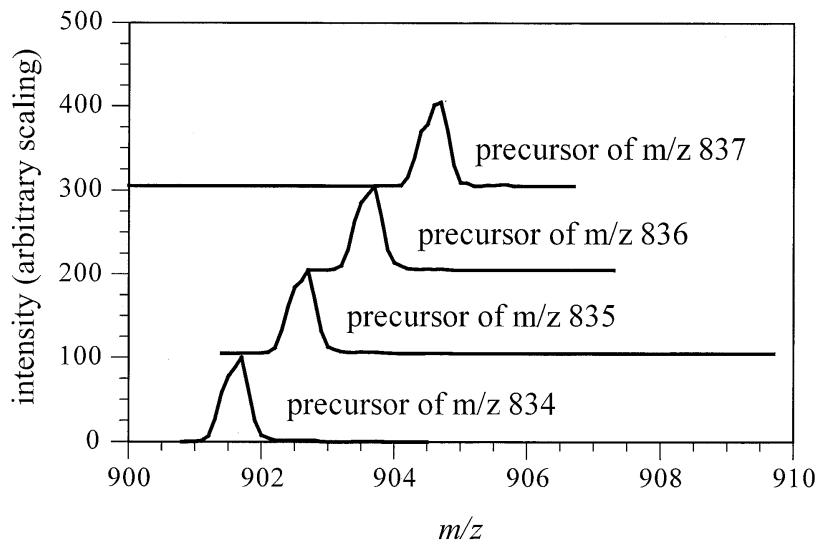

Figure 2. Precursor ion scans for product ions of 32-dimethoxysirolimus, (DMS). Ion intensities are normalized to give comparable peak heights.

small peaks with the theoretical prediction. Unlike product ion scans, precursor ion scans will generally show isotopic structure, even if one selects the monoisotopic product ion for the scan.

\section{Elucidation of Dissociation Pathways}

Figure 4 illustrates results for the dissociation of isotopic peaks of protonated ESA, $\mathrm{C}_{12} \mathrm{H}_{23} \mathrm{O}_{4}^{+}$. As described above, product ion spectra were scanned over three
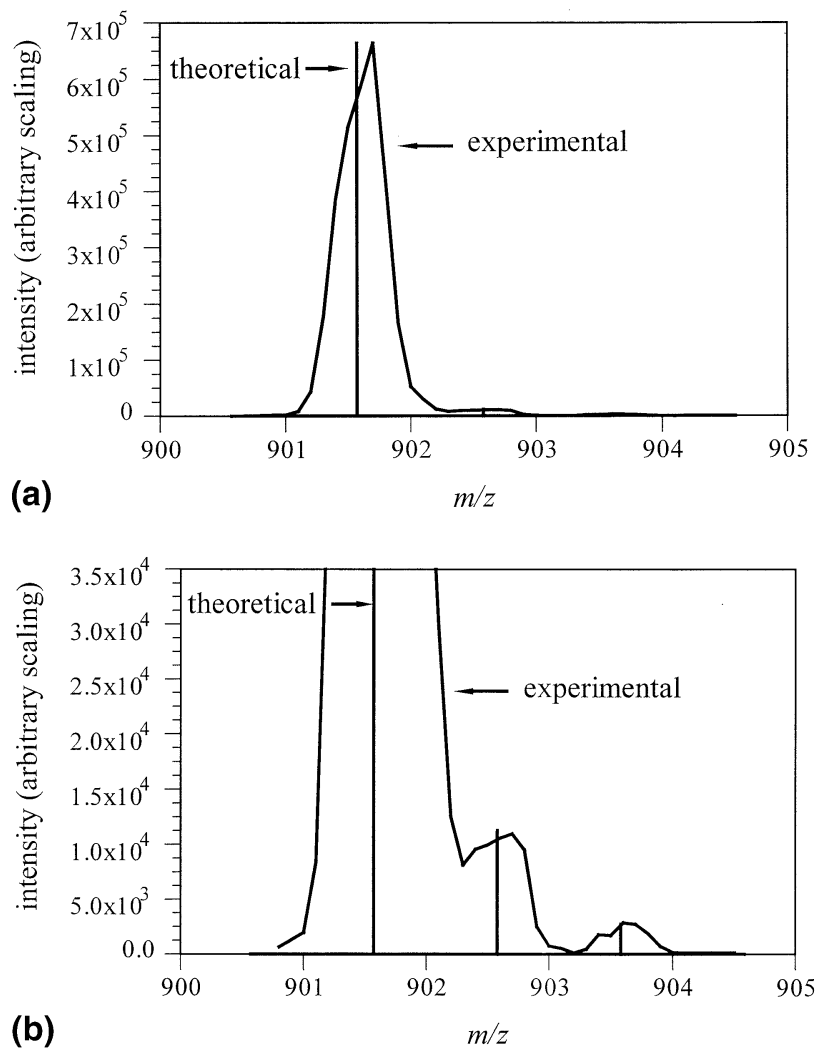

Figure 3. Comparison of theoretical and experimental results for precursor ion scans for $m / z 834$ product ions of DMS. (a) full scale, (b) $20 \times$ blowup of scan.



Figure 4. Experimental results for the dissociation of individual isotopic peaks of protonated di-n-butyl ester of succinic acid $\mathrm{C}_{12} \mathrm{H}_{23} \mathrm{O}_{4}^{+}$, (ESA). Ion intensities are normalized to give comparable peak heights.

regions. The spectra shown are a composite of these three regions. Figure 5 shows a subset of the experimental data taken from Figure 4. The experimental results are shown as profile spectra. The dissociation pathway is assumed to be

$$
\mathrm{C}_{12} \mathrm{H}_{23} \mathrm{O}_{4}{ }^{+} \rightarrow \mathrm{C}_{4} \mathrm{H}_{5} \mathrm{O}_{3}{ }^{+}+\mathrm{C}_{8} \mathrm{H}_{18} \mathrm{O}
$$

Theoretical results in the form of stick spectra are superimposed on the experimental mass spectra. Because of good agreement we conclude that the hypothesized dissociation pathway is "reasonable" in the sense that the results do not exclude this hypothesis.

One can go a step further and use the isotopic results to distinguish between alternative hypothetical dissociation pathways. For example, an alternative hypothesis for the dissociation ESA is

$$
\mathrm{C}_{12} \mathrm{H}_{23} \mathrm{O}_{4}{ }^{+} \rightarrow \mathrm{C}_{5} \mathrm{H}_{9} \mathrm{O}_{2}{ }^{+}+\mathrm{C}_{7} \mathrm{H}_{14} \mathrm{O}_{2}
$$

As shown in Figure 6 this alternative hypothesis yields results that agree poorly with the experiment, so this pathway can be ruled out.

The spectral contrast angle is a good way to compare the similarity between two spectra [21]. For the dissociation of the $(\mathrm{M}+2)^{+}$isotopic peak of ESA the spectral contrast angle between the theoretical results for the two hypothesized dissociation pathways is 10.5 degrees, indicating that the two pathways are clearly distinguishable from each other. Thus, interpretation of isotopic patterns from the dissociation of selected parent ion isotopic peaks may allow one to distinguish between two different isobaric dissociation pathways for the same parent ion.

On the other hand, suppose we ask whether isotopic patterns are useful in ruling out an incorrect hypothesis for parent ion composition. Consider the following hypothetical process, which is isobaric with the dissociation of the protonated di-n-butyl ester of succinic acid. 


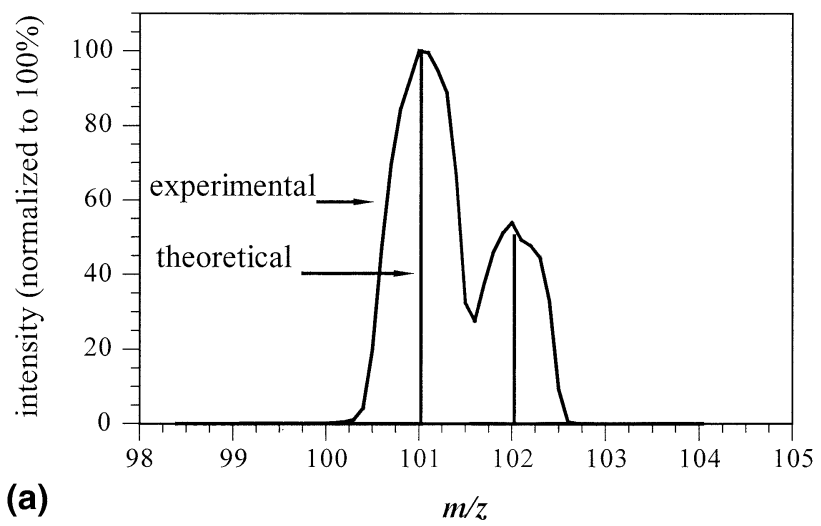

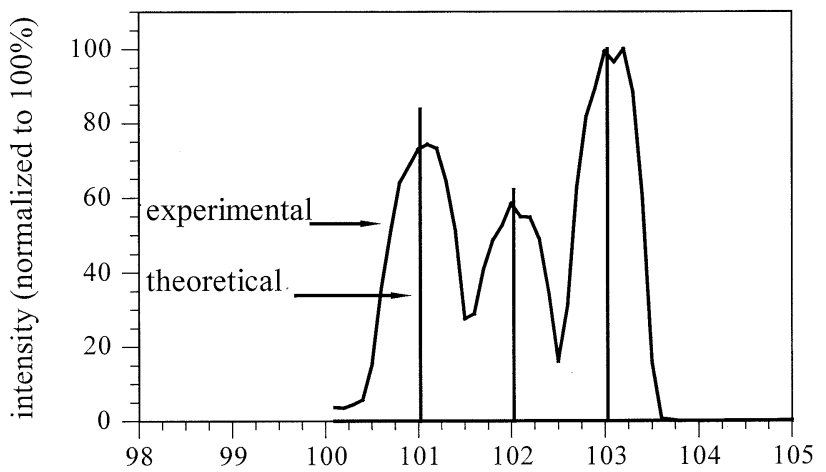

(b)

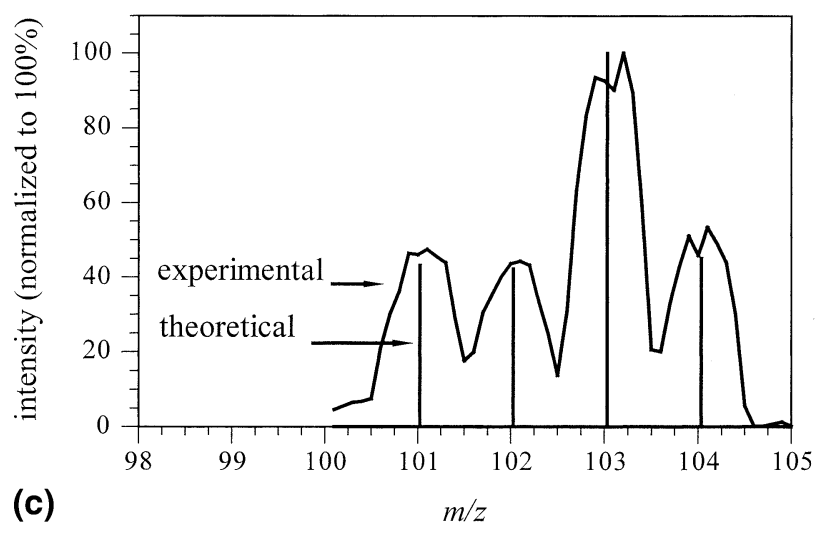

Figure 5. Comparison of theoretical and experimental results for dissociation of individual isotopic peaks of ESA. Experimental data taken as a subset of data in Figure 4. Ion intensities are normalized to give comparable peak heights. The calculation is based on a product ion formula of $\mathrm{C}_{4} \mathrm{H}_{5} \mathrm{O}_{3}^{+}$. Parent ions are $m / z$ (a) 232, (b) 233, and (c) 234 .

$$
\mathrm{C}_{14} \mathrm{H}_{35} \mathrm{~N}_{2}{ }^{+} \rightarrow \mathrm{C}_{6} \mathrm{H}_{15} \mathrm{~N}^{+}+\mathrm{C}_{8} \mathrm{H}_{20} \mathrm{~N}
$$

The experimental result agrees poorly with the theoretical result, as shown in Figure 7 . We therefore conclude that that eq 14 cannot represent the dissociation process.

The examples above deal with three different conceptual cases. The first is to assess the "reasonableness"

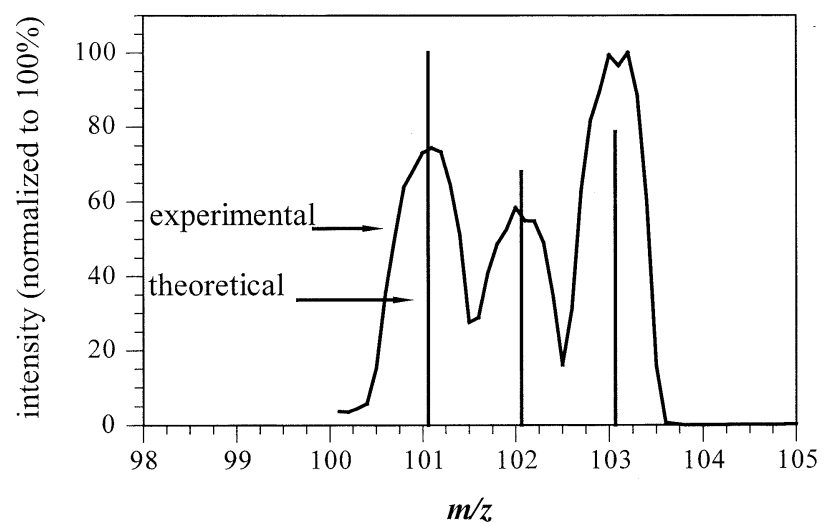

Figure 6. Alternative hypothesized pathway for dissociation of $\mathrm{m} / \mathrm{z} 233$ parent ion of ESA, assuming $\mathrm{C}_{5} \mathrm{H}_{5} \mathrm{O}_{2}^{+}$is the product ion (eq 13). Theoretical results are presented as a stick spectrum. Experimental results are superimposed in the form of a profile spectrum. of a hypothetical dissociation pathway, without comparing it to alternative pathways. The second is to distinguish between two different isobaric dissociation pathways, given that both arise from the same parent ion. The third is to distinguish between two different isobaric dissociation pathways, given that the two arise from ions of differing chemical compositions. We be-

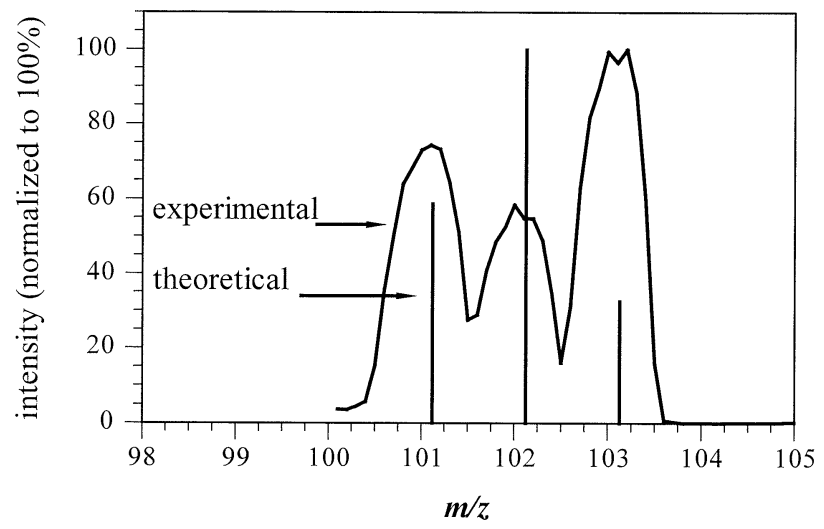

Figure 7. Alternative hypothesized pathway for dissociation of $m / z 233$ parent ion, assuming the parent ion is $\mathrm{C}_{14} \mathrm{H}_{35} \mathrm{~N}_{2}^{+}$and the product ion is $\mathrm{C}_{6} \mathrm{H}_{15} \mathrm{~N}^{+}$(eq 14). Theoretical results are presented as a stick spectrum. Experimental results are superimposed in the form of a profile spectrum. 



Figure 8. Theoretical tandem mass spectrum of $\mathrm{C}_{12} \mathrm{H}_{4}^{79} \mathrm{Br}_{3}^{81} \mathrm{Br}_{3}^{+}(m / z$ 628) through loss of $0-6$ bromine atoms.

lieve that the differences between Figures $5 b, 6$, and 7 is partly attributable to contributions from ${ }^{18} \mathrm{O}$.

\section{Dissociation of Halogenated Hydrocarbons}

Todd et al. measured the isotopic patterns from the dissociation of single isotopic peaks of multiply-halogenated hydrocarbons [4]. We have applied the present theory to one of the systems they studied, the dissociation of the $\mathrm{m} / z 627.5$ isotopic peak $\mathrm{C}_{12} \mathrm{H}_{4} \mathrm{Br}_{6}^{+}$via the loss of one or more bromine atoms. Our calculated results, presented in Figure 8 , agree with their experimental results.

The peaks from the loss of $\mathrm{Br}_{2}$ showed an approximate 1:3:1 intensity ratio for the cluster of product ion peaks, $m / z$ 465.7, 467.7, and 469. Todd et al. argued that this ratio should be strictly 1:3:1, independent of the natural abundance of bromine isotopes, and that the analogous case of dissociation of $\mathrm{C}_{12} \mathrm{H}_{4} \mathrm{Cl}_{6}^{+}$losing two chlorine atoms would produce the same 1:3:1 ratio when dissociating the analogous isotopic peak $(\mathrm{m} / \mathrm{z} 363.8$ in this case). We confirmed by an independent calculation that this speculation is correct (data not shown), provided that one has only the two isotopes of bromine or chlorine to consider. However, if one also considers ${ }^{13} \mathrm{C}$, the ratios deviated from the idealized 1:3:1 ratio. In case of $\mathrm{C}_{12} \mathrm{H}_{4} \mathrm{Br}_{6}^{+}$ the more rigorous theory predicts ratios of 100:301:101 when all isotopic contributions are included.

In some cases the simplified approaches produce much larger relative errors. Consider the dissociation of $\mathrm{Cl}_{12} \mathrm{H}_{4} \mathrm{Br}_{3} \mathrm{Cl}_{3}^{+}$via the loss of $\mathrm{BrCl}$, a system closely related to that discussed above. This case might seem to be a perfect analogue to the dissociation of $\mathrm{C}_{12} \mathrm{H}_{4} \mathrm{Br}_{6}^{+}$ via the loss of $\mathrm{Br}_{2}$, or the dissociation of $\mathrm{C}_{12} \mathrm{H}_{4} \mathrm{Cl}_{6}^{+}$via the loss of $\mathrm{Cl}_{2}$. Consequently, one might expect to observe the same 1:3:1 intensity patterns when dissociating analogous isotopic peaks. However, the correct theoretical ratio is 100:369:102, far different than the 1:3:1 ratio expected from the simplified theory. The difference arises primarily from the differences in the natural abundance of the chlorine isotopes compared to the analogous bromine isotopes.

A possible complicating factor in applying isotopic methods is that elemental isotopic abundances are not constants of nature but vary somewhat, depending on the source of material. This uncertainty in the input data produces uncertainty in the outputs. Although Todd et al. have shown cases where isotopic patterns are not altered by variations in the natural isotopic abundance [4], one can conclude from the discussion above that one should not rely upon this assumption in more complex systems.

\section{Mass Spectral Fingerprinting}

The intensity patterns from the dissociation of single isotopic peaks form a two-dimensional map. One can think of this two-dimensional dissociation map as a fingerprint. As can be seen from the results for ESA, these fingerprints may contain very distinctive patterns. Furthermore, it was shown by the examples in Figures 4-7 that these patterns tend to be characteristic of specific parent/product ion pairs. Consequently, the information-rich spectra from dissociation of selected isotopic peaks are potentially useful for confirmation of compound identity and for aiding the interpretation of molecular structure from MS/MS data. Although there is no guarantee that a compound will dissociate according to some preconceived pathway, if the hypothesized pathway does exist, the theory predicts the branching ratios for the isotopic peaks of the product ions.

This ability to generate the fingerprints from first principles avoids some difficulties associated with most methods of mass spectral fingerprinting. Most mass spectral fingerprinting methods require experimentally generated spectra, which means that one must obtain pure reference compounds to generate a library. This requires that the experimental conditions be defined and reproducible. Otherwise, relative intensities of the different pathways may vary widely enough to prevent finding matches to the library spectra. However, the application of the present method to mass spectral fingerprinting does not necessarily require the development of a library because the isotopic profiles could be calculated "on-the-fly", and they do not depend on experimental conditions. This is somewhat analogous to the use of exact mass measurements for confirmation of chemical composition, where exact masses of pro- 
posed compounds are calculated as needed rather than being stored in libraries.

The isotopic distributions of the product ion are independent of ionization method, collision energy, or type of mass analyzer used, assuming the absence of any significant isotope effect in the dissociation process. Consequently, the results will be reproducible between instruments and can be the basis for a universal method of confirming compound identity and for structural elucidation studies.

A related advantage of using isotopic fingerprinting information for confirmation is that because the fingerprints are independent of experimental conditions, it should be possible to use very tight criteria for matching spectra. Setting tight criteria would, however, require that very high quality data be obtained. For example, if an ion counting data acquisition system is used then the data follow Poisson statistics. Therefore that one must detect at least 10,000 ions to have a $1 \%$ standard deviation in abundance of a peak [22].

The most useful information in the two-dimensional fingerprint will usually be found in the intermediate mass region, at roughly half the mass of the parent ion. This is where the isotopic structure is most complex, and hence the most information-rich. This is also the region where isotopic complexity is most likely to cause interpretation difficulties, and consequently the region in which the present theory will prove most useful. Product ions that are close to the low- and high-mass ends of the product ion spectrum tend to have distributions dominated by a single mass. At the low-mass side the dominant mass is mono-isotopic. At the highmass end the dominant mass is shifted upward in mass from the mono-isotopic mass. The amount of shift equals the approximate difference between the masses of the parent ion isotopic peak selected for dissociation and the mono-isotopic peak of the parent ion. Martin et al. reported this pattern in a related context [2]. They observed the isotopic patterns of daughter ions from incompletely resolved parent ions. Dissociation from both $\mathrm{M}^{+}$and $(\mathrm{M}+1)^{+}$occurred in their experiments. One could use the present theory to quantitatively predict such results by applying a weighted sum of the calculations for the $\mathrm{M}^{+}$and $(\mathrm{M}+1)^{+}$dissociations. A second example of this effect is given in Figure 9, which shows experimental results for a high-mass product formed from the dissociation of DMS. A third example is evident in the dissociation of the brominated compounds discussed in relation to Figure 8 .

Figure 10 shows this same effect for the following idealized dissociation processes:

$$
\begin{aligned}
\mathrm{C}_{40} \mathrm{H}_{84} \mathrm{~N}^{+} & \rightarrow \mathrm{C}_{39} \mathrm{H}_{81} \mathrm{~N}^{+}+\mathrm{CH}_{3} \\
& \rightarrow \mathrm{C}_{20} \mathrm{H}_{43} \mathrm{~N}^{+}+\mathrm{C}_{19} \mathrm{H}_{41} \\
& \rightarrow \mathrm{CH}_{5} \mathrm{~N}^{+}+\mathrm{C}_{39} \mathrm{H}_{79}
\end{aligned}
$$

The middle-mass pathway is more isotopically complex than the light- or heavy-mass pathways.

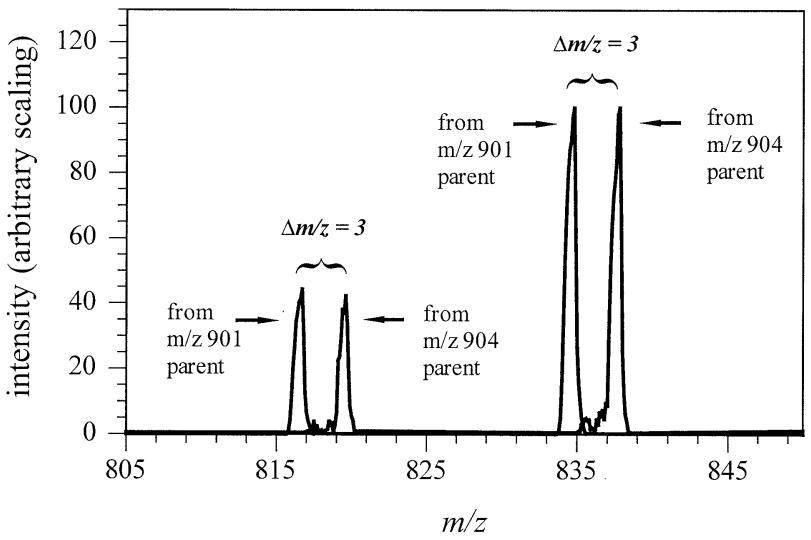

Figure 9. Product ions of two different isotopic peaks of DMS. Ion intensities are normalized to give comparable peak heights.

\section{Tandem Mass Spectra of Mixtures}

Interpretation of tandem mass spectra of samples containing mixtures or impurities can be simplified by the methods presented here. Recently Valianpour et al. presented results on the tandem mass spectrometry of cardiolipins [23]. Cardiolipins are a class of compounds consisting of four fatty acids connected by ester linkages to a core group. The core group consists of three glycerol and two phosphate groups connected via ester linkages. The structure of a generic doubly charged negative cardiolipin ion is presented in Figure 11. In the work of Valianpour et al., the fatty acid groups are designated by a notation such as C18:2, where 18 refers to the carbon chain length, and 2 refers to the number of double bonds in the carbon chain. The parent ion is designated by a notation such as $\left[(\mathrm{C} 18: 2)_{3} /(\mathrm{C} 18: 1)_{1}-\right.$ $2 \mathrm{H}]^{2}-\mathrm{CL}$, which indicates that the parent ion is doubly charged via the loss of two protons, and that is composed of two types of fatty acid groups, three copies of C18:2 and one copy of C18:1. The CL refers to the cardiolipin core. They also used a shortened notation such as $(\mathrm{C} 18: 2)_{3} /(\mathrm{C} 18: 1)_{1}-\mathrm{CL}$ to mean the same thing. For the negative ion composed of a deprotonated fatty acid they used notation such as C18:1. For consistency we will use their notation.

Valianpour et al. speculated that the fatty acids dissociated from the core randomly, and that the intensity of the C18:2 (m/z 279.2) ion should therefore be three times that of the C18:1 $(\mathrm{m} / \mathrm{z} 281.2)$ ion from the dissociation of the mono-isotopic (C18:2) 3 /(C18:1) - CL parent ion. However, rather than the expected 3:1 ratio they observed a ratio of 2.3:1. They suggested that the mono-isotopic $(\mathrm{C} 18: 2)_{3} /(\mathrm{C} 18: 1)_{1}-\mathrm{CL}$ parent ion is likely contaminated by a significant contribution from an isotopic peak of the $(\mathrm{C} 18: 2)_{4}-\mathrm{CL}$ parent ion, and that the contribution from the dissociation of the contaminant ion may lower the ratio from the expected 3:1 value to the observed 2.3:1 ratio.

To test this hypothesis we calculated the expected product ion spectrum using our proposed theory, together with the assumption of random dissociation 




Figure 10. Theoretical isotopic profiles from low- middle- and high-mass dissociation products for a hypothetical the dissociation process given in eq 15 .

given by Valianpour et al. First we calculated the isotopic distributions of the $(\mathrm{C} 18: 2)_{3} /(\mathrm{C} 18: 1)_{1}-\mathrm{CL}$ and $(\mathrm{C} 18: 2)_{4}-\mathrm{CL}$ parent ions. Then we calculated a weighted sum of these so as to reproduce the first three isotopic peaks of the cluster of parent ions containing $(\mathrm{C} 18: 2)_{4}-\mathrm{CL}$ and $(\mathrm{C} 18: 2)_{3} /(\mathrm{C} 18: 1)_{1}-\mathrm{CL}$. The relative weight factors for these two were 1.000 and 0.934 , respectively. We then calculated a composite isotopic profile for the dissociation of the parent ion whose mass corresponds to the mono-isotopic $(\mathrm{C} 18: 2)_{3} /(\mathrm{C} 18: 1)_{1}-$ $\mathrm{CL}$ parent ion. Because the sample is a mixture, this peak also includes the contribution from an isotopic peak of the $(\mathrm{C} 18: 2)_{4}-\mathrm{CL}$ parent ion. The result is a predicted intensity ratio for the $m / z 279.2$ and 281.2 peaks of 3.8:1, significantly higher than the $3: 1$ ratio they originally expected and much higher than the 2.3:1 ratio experimentally observed. Therefore, it is not likely that isotopic profiles are responsible for the experimentally observed ratio and one must look elsewhere for a better explanation. The most obvious possibility is that the fatty acids do not dissociate randomly, but that there is a preferential effect that depends on the structures of the fatty acid groups.

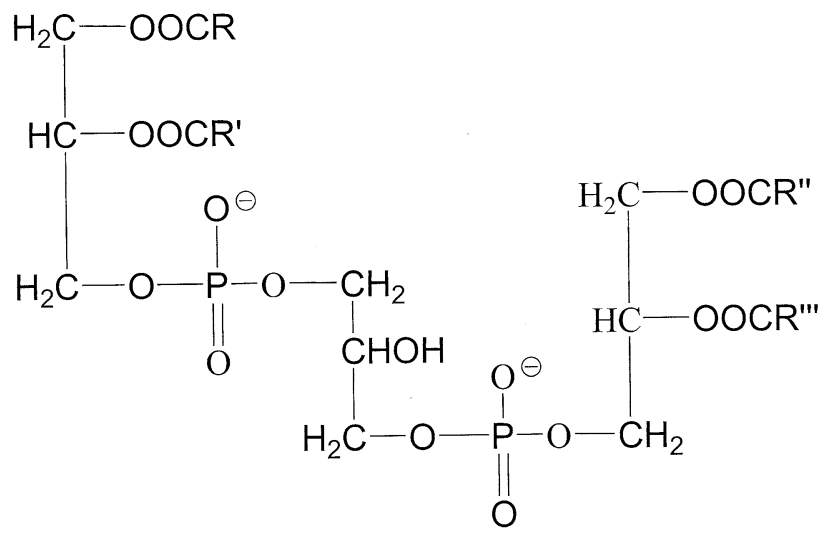

Figure 11. Generic structure of cardiolipin. Structure is shown as the doubly-charged negative ion.
Isotopic Assignment of Large Molecules

An interesting application of using isotopic information in tandem mass spectrometry is in the isotopic assignment in large-molecule mass spectra by using the dissociation of selected isotopic peaks. For this application, it was previously shown that variations of elemental isotopic abundances were not a significant factor in making the mono-isotopic assignment [5]. The theoretical discussion given in that paper was based on simplified assumptions, namely that the chemical elements in the formula each contain only two isotopes, and that the elemental isotopes are spaced one $\mathrm{Da}$ apart. While that treatment yielded useful results, it would be preferable to use an exact theory rather than an approximation.

\section{Computational Efficiency}

Using an ultrahigh speed algorithm [11] the vectors $g$ and $f$ can be easily computed in a small fraction of a second. The calculation of the outer product (the Cmatrix) is also very efficient. We used Psi-Plot to calculate the outer products for the examples in this paper, and the results were produced without any perceptible delay [24]. A compiled version would have been even more efficient.

\section{Weak Mono-Isotopic Peaks}

It was noted above that a potential application of the theory relates to cases where the mono-isotopic parent ion is not the base peak in the parent ion spectrum. This typically occurs for peptides at molecular weights of 2000-3000 Da or higher [2]. For example, Figure 12 shows a calculated parent ion isotopic distributions for a hypothetical "averagine 22 " and "averagine ${ }_{44}$ " peptides [25] of formulas $\mathrm{C}_{109} \mathrm{H}_{171} \mathrm{~N}_{30} \mathrm{O}_{33} \mathrm{~S}^{+}$and $\mathrm{C}_{218} \mathrm{H}_{342} \mathrm{~N}_{60} \mathrm{O}_{66} \mathrm{~S}_{2}^{+}$. The respective mono-isotopic molecular weights are 2460 and 4920 , and the respective mono-isotopic peak intensi- 


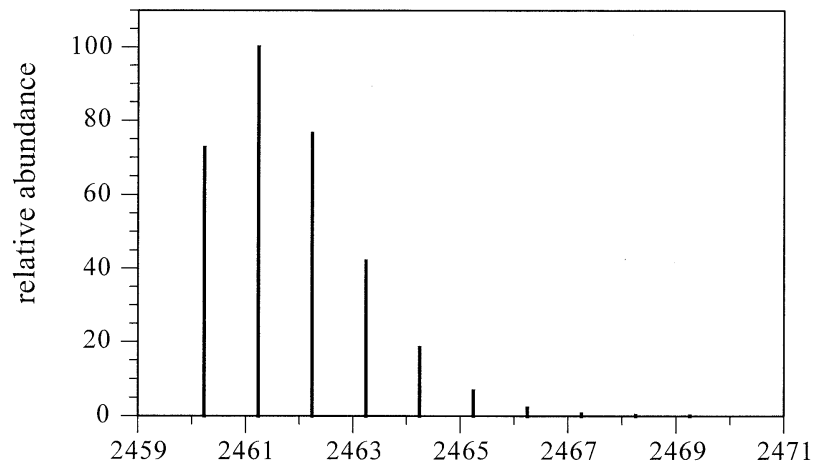

(a)



(b)

$m / z$

Figure 12. Theoretical isotopic distributions of "averagine ${ }_{22}$ " $\left(\mathrm{C}_{109} \mathrm{H}_{171} \mathrm{~N}_{30} \mathrm{O}_{33} \mathrm{~S}^{+}\right)$and "averagine ${ }_{44}$ " $\left(\mathrm{C}_{218} \mathrm{H}_{342} \mathrm{~N}_{60} \mathrm{O}_{66} \mathrm{~S}_{2}^{+}\right)$.

ties are 73 and $25 \%$ of the base peak. For some classes of compounds the mono-isotopic peak ceases to be dominant at lower molecular weight. Transition metal chemistry provides a particularly rich source of examples. Wolf et al. have shown electrospray mass spectra of an organonickel ion whose mono-isotopic peak at $\mathrm{m} / \mathrm{z} 345$ was only $\sim 13 \%$ of the base peak at $\mathrm{m} / \mathrm{z} 347$ [26]. A more extreme case is presented by the ion $\mathrm{C}_{88} \mathrm{H}_{64} \mathrm{P}_{4} \mathrm{Cl}_{5} \mathrm{Ru}_{2}^{-}$which was studied by James et al. [27]. For this ion we calculate that the intensity of the mono-isotopic peak at $\mathrm{m} / \mathrm{z} 1611$ is only $0.24 \%$ of the base peak at $\mathrm{m} / \mathrm{z} 1625$. Multiplyhalogenated compounds are another rich source of examples. The mono-isotopic parent ion of $\mathrm{C}_{12} \mathrm{H}_{4} \mathrm{Br}_{6}^{+}$ compound discussed above has a theoretical intensity of only $5 \%$ of the base peak.

\section{Generalizations and Additional Implications}

The simplest generalization is to relax the requirement that there is only a single dissociation pathway. If multiple dissociation pathways exist one would simply perform separate calculations for each pathway and then sum the results, weighted according to the branching ratios of the pathways.

Exact masses of product ions are easily grafted onto the theory, assuming exact masses are known for the isotopic peaks of product ion. One simply substitutes the exact mass for $D(k-n, n)$ in eq 5 .

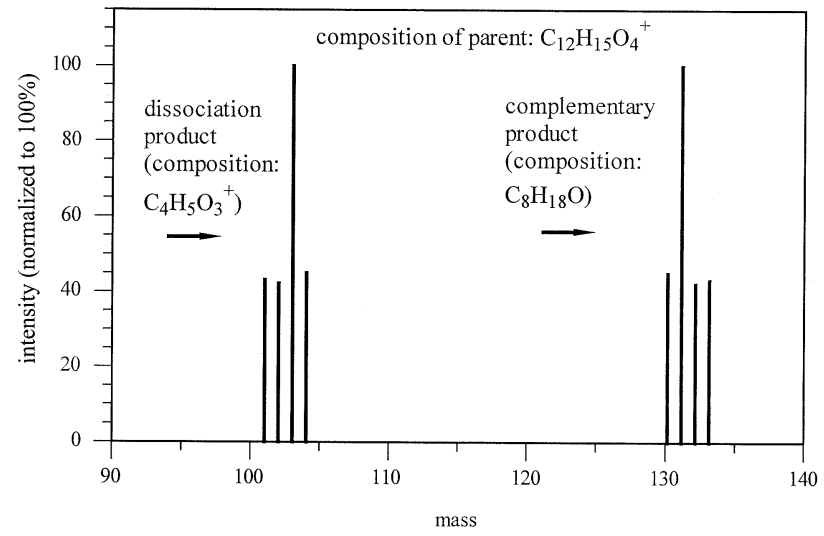

Figure 13. Mirror image property for product ion and complementary product, calculated results for the dissociation of protonated di-n-butyl ester of succinic acid $\mathrm{C}_{12} \mathrm{H}_{23} \mathrm{O}_{4}^{+}$, (ESA).

The theory presented in this paper applies to any number of MS/MS stages. For example, if one were to select a single isotopic peak of the parent ion for dissociation, and then select a single isotopic peak of the product ion for another stage of dissociation, the isotopic distribution that would result can be accurately predicted by the presented method.

The proposed theory initially assumed that one knows the chemical formulas of the parent ion, product ion, and complementary product. However, such knowledge is not necessary. For example, it can be shown that if one knows the chemical composition of the product ion, together with an experimental measurement of the isotopic profile of the parent ion, one can generate the isotopic distribution of the complementary product using a deconvolution procedure [28]. One may then apply the present algorithm.

Isotopically-labeled compounds require special consideration. This case is handled by defining a fictitious element. For example, if a particular position in the molecule were labeled with $90 \%$ pure ${ }^{13} \mathrm{C}$, one would define a fictitious element, $X$, composed of $10 \%$ mass 12 and $90 \%$ mass 13 . The chemical formula of the compound would then be altered to account for the fictitious element. The algorithm is then applied to the modified chemical formula. This requires knowing the fate of the fictitious element during the dissociation process, specifically whether the labeled position contributes to the dissociation product or to the complementary product.

One consequence of the algorithm is that the distributions for the two complementary products are mirror images of each other. A calculated example is shown in Figure 13 . This mirror-image property arises because the roles of the product ion and complementary product can be switched by transposing the $C$-matrix. The mirror image property was noted previously, based on a simpler, more restricted theory [4]. However, it can be shown that mirror image property is rigorously true in all cases. 


\section{Conclusions}

The present paper develops a general theory for predicting the isotopic distributions resulting from the dissociation of single isotopic peaks. The algorithm uses the outer product of the full isotopic distributions of the product ion and complementary product, which generates a " $\mathrm{C}$ matrix". The intensity patterns for the isotopic distribution are contained in the reverse diagonal bands of this matrix. The isotopic profiles from the dissociation of single isotopic peaks are potentially useful for confirmation of compound identity and for aiding chemical structure characterization by MS/MS because they are information-rich, independent of instrument type, and independent of ionization technique. In addition, the method is potentially useful for tandem mass spectrometry applications of large molecules in which the mono-isotopic peak is too weak to produce a strong product ion signal. The theory provides a new and useful tool to aid in the interpretation of the complicated isotopic structure that can result from such experiments.

\section{References}

1. Martin, S. A.; Biemann, K. A Comparison of $\mathrm{keV}$ Atom Bombardment Mass Spectra of Peptides Obtained with a Two-Sector Mass Spectrometer with Those from a Four-Sector Tandem Mass Spectrometer. Int. J. Mass Spectrom. Ion Processes 1987, 78, 213-228.

2. Martin, S. A.; Scoble, H. A.; Costello, C. E.; Biemann, K. Resolution Reqirements of a High Performance Tandem Mass Spectrometer. Proceedings of the 35th ASMS Conference; Denver, CO, May 1987, pp 78-79.

3. Bozorgzadeh, M. H.; Morgan, R. P.; Beynon, J. H. Application of Mass-analyzed Ion Kinetic Energy Spectrometry (MIKES) to the Determination of the Structures of Unknown Compounds. Analyst 1978, 103, 613-622.

4. Todd, P. J.; Barbalas, M. P.; McLafferty, F. W. Collisional Activation Mass Spectra of Ions Containing Polyisotopic Elements. Org. Mass Spectrom. 1982, 17, 79-80.

5. O'Conner, P. B.; Little, D. P.; McLafferty, F. W. Isotopic Assignment in Large-Molecule Mass Spectra by Fragmentation of a Selected Isotopic Peak. Anal. Chem. 1996, 68, 542-545.

6. Roussis, S. G. Automated Tandem Mass Spectrometry by Orthogonal Acceleration TOF Data Acquisition and Simultaneous Magnet Scanning for the Characterization of Petroleum Mixtures. Anal. Chem. 2001, 73, 3611-3623.

7. Barbalas, M. P.; McLafferty, F. W.; Occolowitz, J. L. Targeted Analysis of $\beta$-Lactam Antibiotics by Tandem Mass Spectrometry. Biomed. Mass Spectrom. 1983, 10, 258-261.

8. Hunt, D. F.; Shabanowitz, J. Determination of Organosulfur Compounds in Hydrocarbon Mixtures by Collisional Activated Dissociation Mass Spectrometry. Anal. Chem. 1982, 54, $574-578$.
9. Palmer, P. T.; Enke, C. G. Empirical Formula Determination by Triple Quadrupole Mass Spectrometry. Proceedings of the 34th ASMS Conference; Cincinnati, OH, June 1986, pp 288-289.

10. Tou, J. C.; Zakett, D.; Caldecourt, V. J. Tandem Mass Spectrometry of Industrial Chemicals. In Tandem Mass Spectrometry; McLafferty, F. W., Ed.; John Wiley and Sons: New York, 1983; pp 441-446.

11. Rockwood, A. L.; Van Orden, S. L. Ultrahigh-Speed Calculation of Isotope Distributions. Anal. Chem. 1996, 68, 2027-2030.

12. Rockwood, A. L.; Van Orden, S. L.; Smith, R. D. Rapid Calculation of Isotope Distributions. Anal. Chem. 1995, 67, 2699-2704.

13. Kubinyi, H. Calculation of Isotope Distributions in Mass Spectrometry. A Trivial Solution for a Non-Trivial Problem. Anal. Chim. Acta 1991, 247, 107-119.

14. Senko, M. W. IsoPro Version 3.03a, 1998.

15. Yergey, J. A. A General Approach to Calculating Isotopic Distributions for Mass Spectrometry. Int. J. Mass Spectrom. Ion Phys. 1983, 52, 337-349.

16. Hsu, C. S. Diophantine Approach to Isotopic Abundance Calculations. Anal. Chem. 1984, 56, 1356-1361.

17. Brownawell, M. L.; San Filippo, J., Jr. A Program for the Synthesis of Mass Spectral Isotopic Abundances. J. Chem. Edu. 1982, 59, 663-665.

18. Werlen, R. C. Effect of Resolution on the Shape of Mass Spectra of Proteins: Some Theoretical Considerations. Rapid Commun. Mass Spectrom. 1994, 8, 976-980.

19. Parker, S. P. (Ed.) Dictionary of Mathematics; McGraw-Hill, New York: 1997, p 177.

20. Emmanuel C. I.; Barrie, W. J. Digital Signal Processing, a Practical Approach, 2nd ed.; Prentice Hall: New York, 2002; p 5.

21. Wan, K. X.; Vidavsky, I.; Gross, M. L. Comparing Similar Spectra: From Similarity Index to Spectral Contrast Angle. J. Am. Soc. Mass Spectrom. 2001, 13, 85-88.

22. Maksodian Y. L. Probability and Statistics with Applications; International Textbook Company: Scranton, 1966; pp 83-84.

23. Valianpour, F.; Wanders, R. J. A.; Barth, P. G.; Overmars, H.; van Gennip, A. H. Quantitative and Compositional Study of Cardiolipin in Platelets by Electrspray Ionization Mass Spectrometry: Applications for the Identification of Barth Wyndrome Patients. Clin. Chem. 2002, 48, 1391-1397.

24. Psi-Plot Version 6.5 for Windows; Poly Software International Inc., Pearl River, NY.

25. Senko, M. W.; Beu, S. C.; McLafferty, F. W. Determination of Monoisotopic Masses and Ion Populations for Large Biomolecules from Resolved Isotopic Distributions. J. Am. Soc. Mass Spectrom. 1995, 6, 229-233.

26. Wolf, B. P.; Sumner, C. A.; Grapperhaus, C. A., Musie, G.; Darensbourg, M. Y.; Russell, D. H. ESI-MS of Organometalic Compounds. Proceedings of the 44th ASMS Conference; Portland, OR, May 1996, p 659.

27. James, B.; Stevens, K. L.; Buzard, D. J.; Keith, J. M.; Pavlovich, J. G.; Lipshutz, B. H. Analysis of Copper and Ruthenium Complexes by ESI-MS. Proceedings of the 44th ASMS Conference; Portland, OR, May 1996, p 661.

28. Rockwood, A. L. Deconvoluting Isotopic Distributions to Evaluate Parent/Fragment Ion Relationships. Rapid Commun. Mass Spectrom. 1997, 11, 241-248. 